Gazi University
Journal of Science
http://dergipark.gov.tr/gujs

\title{
Generalized Regression-Cum-Exponential Estimators Using Two Auxiliary Variables for Population Variance in Simple Random Sampling
}

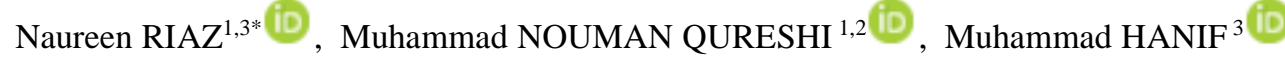 \\ ${ }^{I}$ National College of Business Administration \& Economics, Department of Statistics, Lahore, Pakistan \\ ${ }^{2}$ University of Minnesota, School of Statistics, Minneapolis-55108, USA \\ ${ }^{3}$ Lahore Garrison University, Department of Statistics, Lahore, Pakistan
}

\section{Highlights}

- Generalized regression-cum-exponential type estimators are proposed to estimate population variance.

- Bias and MSE have been derived for the proposed estimators.

- Simulation and empirical study proves better functioning of the proposed estimators than the existing one.

\begin{tabular}{l} 
Article Info \\
Received: 01/06/2018 \\
Accepted: $24 / 10 / 2018$ \\
Keywords \\
\hline Auxiliary Information \\
Exponential Estimators \\
Percent Relative \\
Efficiency
\end{tabular}

\section{INTRODUCTION}

Auxiliary information is commonly used to improve the efficiency of population parameters of interest. History filled with a lot of authors that have used auxiliary information in order to get the prices estimates. Classical ratio, product, and regression and their mixture-type of estimators are good examples and cornerstone in this background. The estimation of population variance considered to be the best measure in the variations of study variable. Various authors have been done work on estimating the population variance. [1] introduced classical ratio and regression estimators in finite population variance. The effort of [2,3] may be well-thought-out as an early effort in the estimation of population variance.[4] developed the exponential ratio and exponential product type estimators in population variance.[5] developed generalized exponential estimator. [6] developed a ratio-type estimator for finite population variance. [7] utilized a single auxiliary variable to propose an estimator in population variance and provide more efficient results as compare to the ratio estimator. The studies related to the estimation of population variance have made by different authors such as [8-22].

After a brief introduction and literature review, some basics notations and existing estimators are mentioned in Section 2. The expressions of approximate bias and MSE of the proposed estimators are derived in Section 3. Section 4 is based on efficiency comparisons. A numerical study is illustrated in Section 5 on three real populations. Conclusion and remarks are given in Section 6. 


\section{NOTATION AND VARIOUS EXISTING ESTIMATORS}

Let $U=\left(U_{1}, U_{2}, \ldots, U_{N}\right)$ having the units for a finite population $U$ for the population size $N$ and a sample of size $n$ is selected from the population by using the method of $S R S$ without replacement. Here $S_{y}^{2}, S_{x}^{2}$ and $S_{z}^{2}$ be the population variances, while $s_{y}^{2}, s_{x}^{2}$ and $s_{z}^{2}$ be the sample variance for our study and auxiliary variables respectively. Let, $e_{0}, e_{1}$ and $e_{2}$ be the sampling errors such that:

$$
\begin{aligned}
& S_{y}^{2}=S_{y}^{2}\left(1+e_{0}\right), s_{x}^{2}=S_{x}^{2}\left(1+e_{1}\right), s_{z}^{2}=S_{z}^{2}\left(1+e_{2}\right), \\
& E\left(e_{0}\right)=E\left(e_{1}\right)=E\left(e_{2}\right)=E\left(e_{11}\right)=E\left(e_{22}\right)=0, \\
& E\left(e_{0}^{2}\right)=\theta\left(\lambda_{400}-1\right), E\left(e_{1}^{2}\right)=\theta\left(\lambda_{040}-1\right), E\left(e_{2}^{2}\right)=\theta\left(\lambda_{004}-1\right), \\
& E\left(e_{0} e_{1}\right)=\theta\left(\lambda_{220}-1\right), E\left(e_{0} e_{2}\right)=\theta\left(\lambda_{202}-1\right), E\left(e_{2} e_{1}\right)=\theta\left(\lambda_{022}-1\right), \\
& \rho_{y x}=\frac{S_{y x}}{S_{y} S_{x}}, \rho_{y z}=\frac{S_{y z}}{S_{y} S_{z}}, C_{y}=\frac{S_{y}}{\bar{Y}}, C_{x}=\frac{S_{x}}{\bar{X}}, C_{z}=\frac{S_{z}}{\bar{Z}}, w_{1}=\frac{\alpha_{1}}{\delta_{1}}, w_{2}=\frac{\alpha_{2}}{\delta_{2}} .
\end{aligned}
$$

Let $\bar{Y}, \bar{X}$ and $\bar{Z}$ be the population means whereas $\bar{y}, \bar{x}$ and $\bar{z}$ be the sample means for the study and auxiliary variables respectively. Let $e_{11}, e_{22}$ be the sampling error such that

$$
\begin{aligned}
& \bar{x}=\bar{X}\left(1+e_{11}\right), \bar{z}=\bar{Z}\left(1+e_{22}\right), s_{x}^{2}=S_{x}^{2}\left(1+e_{11}\right), s_{z}^{2}=S_{z}^{2}\left(1+e_{22}\right), \\
& E\left(e_{11}^{2}\right)=\theta C_{x}^{2}, E\left(e_{22}^{2}\right)=\theta C_{z}^{2}, E\left(e_{0} e_{11}\right)=\theta \lambda_{210} C_{x}, E\left(e_{0} e_{22}\right)=\theta \lambda_{201} C_{z}, \\
& E\left(e_{2} e_{11}\right)=\theta \lambda_{012} C_{x}, E\left(e_{1} e_{22}\right)=\theta \lambda_{021} C_{z}, E\left(e_{11} e_{22}\right)=\theta \rho_{x z} C_{x} C_{z}, \theta=\frac{1}{n}, \\
& \lambda_{a b c}=\frac{\mu_{a b c}}{\mu_{200}^{\frac{a}{2}} \mu_{020}^{\frac{b}{2}} \mu_{002}^{c}}, \mu_{a b c}=\frac{1}{N-1} \sum_{i=1}^{N}\left(Y_{i}-\bar{Y}\right)^{a}\left(X_{i}-\bar{X}\right)^{b}\left(Z_{i}-\bar{Z}\right)^{c}, \\
& { }_{y} f_{004}=\frac{\left(\lambda_{004}-1\right)}{\left(\lambda_{400}-1\right)},{ }_{y} f_{220}=\frac{\left(\lambda_{220}-1\right)}{\left(\lambda_{400}-1\right)},{ }_{x} f_{220}=\frac{\left(\lambda_{220}-1\right)}{\left(\lambda_{040}-1\right)}, \\
& { }_{x} f_{022}=\frac{\left(\lambda_{022}-1\right)}{\left(\lambda_{040}-1\right)}, f_{202}=\frac{\left(\lambda_{202}-1\right)}{\left(\lambda_{004}-1\right)},{ }_{z} f_{022}=\frac{\left(\lambda_{022}-1\right)}{\left(\lambda_{004}-1\right)}, \\
& M_{1}=\frac{1}{A^{2}}\left[{ }_{y} f_{004} R_{1}+{ }_{y} f_{040} R_{2}+2{ }_{y} f_{002} R_{3}\right]-\frac{2}{A} R_{4}, P=\frac{\lambda_{012}^{2} R_{2}}{B^{2}\left(\lambda_{004}-1\right)}-\frac{2 R_{3}}{B}-\frac{1}{4} C_{x}^{2}+\lambda_{210} C_{x}, \\
& M_{2}=\frac{\lambda_{012}^{2} R_{2}}{B^{2}\left(\lambda_{004}-1\right)}+\frac{1}{B^{2}}\left(R_{3}-2 \lambda_{012}^{2} \lambda_{210}^{2}\right)-\frac{2 R_{3}}{B}, A=1-{ }_{x} f_{220} f_{022}, B=\left(\frac{1-\lambda_{012}^{2}}{\left(\lambda_{004}-1\right)}\right),
\end{aligned}
$$

where $a, b$ and $c$ be the non-negative integers. The quantities $\mu_{200}, \mu_{020}$ and $\mu_{002}$ be the second order moments and $\lambda_{a b c}$ be the moment ratio.

The sample means and variances of the study and auxiliary variable may obtained as:

$$
\begin{aligned}
& \bar{y}=\frac{1}{n} \sum_{i=1}^{n} y_{i}, \bar{x}=\frac{1}{n} \sum_{i=1}^{n} x_{i} \text { and } \bar{z}=\frac{1}{n} \sum_{i=1}^{n} z_{i}, \\
& s_{y}^{2}=\frac{1}{n-1} \sum_{i=1}^{n}\left(y_{i}-\bar{y}\right)^{2}, s_{x}^{2}=\frac{1}{n-1} \sum_{i=1}^{n}\left(x_{i}-\bar{x}\right)^{2} \text { and } s_{z}^{2}=\frac{1}{n-1} \sum_{i=1}^{n}\left(z_{i}-\bar{z}\right)^{2} .
\end{aligned}
$$

Similarly, the population means and variances of the study and auxiliary variables may be obtained as: 


$$
\begin{aligned}
& \bar{Y}=\frac{1}{N} \sum_{i=1}^{N} Y_{i}, \bar{X}=\frac{1}{N} \sum_{i=1}^{N} X_{i} \text { and } \bar{Z}=\frac{1}{N} \sum_{i=1}^{N} Z_{i}, \\
& S_{y}^{2}=\frac{1}{N-1} \sum_{i=1}^{N}\left(Y_{i}-\bar{Y}\right)^{2}, S_{x}^{2}=\frac{1}{N-1} \sum_{i=1}^{N}\left(X_{i}-\bar{X}\right)^{2} \text { and } S_{z}^{2}=\frac{1}{N-1} \sum_{i=1}^{N}\left(Z_{i}-\bar{Z}\right)^{2} .
\end{aligned}
$$

In literature, the unbiased variance estimator without having auxiliary information for the finite population is

$t_{0}=s_{y}^{2}=\frac{1}{n-1} \sum_{i=1}^{n}\left(y_{i}-\bar{y}\right)^{2}$.

The variance of $t_{0}$ is given as

$$
\operatorname{Var}\left(t_{0}\right)=\theta S_{y}^{4}\left(\lambda_{400}-1\right)
$$

[1] proposed the classical ratio and regression estimators for the estimation of finite population variance as

$$
\begin{aligned}
& t_{1}=s_{y}^{2}\left(\frac{S_{x}^{2}}{s_{x}^{2}}\right), \\
& t_{2}=s_{y}^{2}+b_{1}\left(S_{x}^{2}-s_{x}^{2}\right),
\end{aligned}
$$

where $b_{1}=S_{y}^{2}\left(\lambda_{220}-1\right) / S_{x}^{2}\left(\lambda_{040}-1\right)$, which is regression coefficient.

The expressions of MSE of $t_{1}$ and $t_{2}$ up to the first order approximation are respectively given as

$$
\begin{aligned}
& \operatorname{MSE}\left(t_{1}\right) \approx \theta S_{y}^{4}\left[\lambda_{400}+\lambda_{040}-2 \lambda_{220}\right], \\
& \operatorname{MSE}\left(t_{2}\right) \approx \theta S_{y}^{4}\left(\lambda_{400}-1\right)\left[1-{ }_{x} f_{220} f_{220}\right] .
\end{aligned}
$$

The traditional regression estimator for population variance using the mean of the auxiliary variable is

$$
t_{3}=s_{y}^{2}+b_{2}(\overline{\mathrm{X}}-\bar{x})
$$

where, $b_{2}=S_{y}^{2}\left(\lambda_{210}\right) / \bar{X} C_{x}$. which is regression coefficient.

The expression of mean square error of $t_{3}$ up to the first order approximation is given as

$$
\operatorname{MSE}\left(t_{3}\right) \approx \theta \mathrm{S}_{y}^{4}\left[\left(\lambda_{400}-1\right)-\lambda_{210}^{2}\right] .
$$

[4] introduced exponential ratio and exponential product-type estimators using the information of single auxiliary variable for the finite population variance are

$$
\begin{aligned}
& t_{4}=s_{y}^{2} \exp \left(\frac{S_{x}^{2}-s_{x}^{2}}{S_{x}^{2}+s_{x}^{2}}\right), \\
& t_{5}=s_{y}^{2} \exp \left(\frac{s_{z}^{2}-S_{z}^{2}}{S_{z}^{2}+s_{z}^{2}}\right) .
\end{aligned}
$$


The expressions of $M S E$ for $t_{4}$ and $t_{5}$ up to the first order approximation are respectively given as

$$
\begin{aligned}
& \operatorname{MSE}\left(t_{4}\right) \approx \theta S_{y}^{4}\left[\lambda_{400}+\frac{\lambda_{040}}{4}-\lambda_{220}+\frac{1}{4}\right], \\
& \operatorname{MSE}\left(t_{5}\right) \approx \theta S_{y}^{4}\left[\lambda_{400}+\frac{\lambda_{004}}{4}+\lambda_{202}-\frac{9}{4}\right] .
\end{aligned}
$$

[5] introduced a generalized exponential-type estimator using the auxiliary information of finite population variance as

$t_{6}=s_{y}^{2} \exp \left[\frac{S_{x}^{2}-s_{x}^{2}}{S_{x}^{2}+(\alpha-1) s_{x}^{2}}\right]$.

The expression of minimum $M S E$ of $t_{6}$ up to the first order approximation is

$\operatorname{MSE}_{\min }\left(t_{6}\right)=\theta S_{y}^{4}\left(\lambda_{400}-1\right)\left[1-{ }_{y} f_{220} f_{220}\right]$,

where $\alpha_{\text {opt }}=\left(\lambda_{040}-1\right) /\left(\lambda_{220}-1\right)$.

[8] introduced exponential ratio and exponential product-type estimators using the mean of the auxiliary variable as

$$
\begin{aligned}
& t_{7}=s_{y}^{2} \exp \left(\frac{\bar{X}-\bar{x}}{\bar{X}+\bar{x}}\right), \\
& t_{8}=s_{y}^{2} \exp \left(\frac{\bar{x}-\bar{X}}{\bar{X}+\bar{x}}\right) .
\end{aligned}
$$

The expressions of $M S E$ of $t_{7}$ and $t_{8}$ up to the first order term are given as

$$
\begin{aligned}
& \operatorname{MSE}\left(t_{7}\right) \approx \theta S_{y}^{2}\left[\left(\lambda_{400}-1\right)-\lambda_{210} C_{x}+\frac{1}{4} C_{x}^{2}\right] . \\
& \operatorname{MSE}\left(t_{8}\right) \approx \theta S_{y}^{2}\left[\left(\lambda_{400}-1\right)+\lambda_{210} C_{x}+\frac{1}{4} C_{x}^{2}\right] .
\end{aligned}
$$

[9] suggested new variance estimator as

$$
t_{9}=\frac{s_{y}^{2}}{2}\left[\frac{S_{x}^{2}}{s_{x}^{2}}+\exp \left(\frac{S_{x}^{2}-s_{x}^{2}}{S_{x}^{2}+s_{x}^{2}}\right)\right] .
$$

The expression of $M S E$ of $t_{9}$ up and around to the first order term is given as

$$
\operatorname{MSE}\left(t_{9}\right) \approx \theta S_{y}^{4}\left[\left(\lambda_{400}-1\right)+\frac{3\left(\lambda_{040}-1\right)}{16}\left(3-8_{x} f_{220}\right)\right]
$$


[10] suggested a new exponential-type estimator. The estimator along with the expression of its variance is

$$
\begin{aligned}
& t_{10}=s_{y}^{2} \exp \left[\frac{\sqrt{\bar{X}}-\sqrt{\bar{x}}}{\sqrt{\bar{X}}+\sqrt{\bar{x}}}\right], \\
& \operatorname{MSE}\left(t_{10}\right)=\theta S_{y}^{4}\left[\lambda_{400}+\frac{1}{2} C_{x}\left(\frac{1}{8} C_{x}-\lambda_{210}\right)-1\right] .
\end{aligned}
$$

[11] proposed exponential-type estimator using a single auxiliary variable as

$t_{11}=\lambda s_{y}^{2} \exp \left[\frac{\mathrm{c}(\overline{\mathrm{X}}-\bar{x})}{\bar{X}+(\mathrm{d}-1) \overline{\mathrm{x}}}\right] \exp \left[\frac{\mathrm{e}\left(S_{x}^{2}-s_{x}^{2}\right)}{S_{x}^{2}+(\mathrm{f}-1) s_{x}^{2}}\right]$,

the expression of minimum mean square error of $t_{11}$ up to the first order approximation is given as

$\operatorname{MSE}_{\text {min }}\left(t_{11}\right)=\theta S_{y}^{4}\left[\left(\lambda_{400}-1\right)-\lambda_{210}^{2}-\left\{\frac{\left(\left(\lambda_{220}-1\right)-\lambda_{210}\left(\lambda_{030}\right)\right)^{2}}{\left(\lambda_{040}-1\right)-\lambda_{030}^{2}}\right\}\right]$.

\section{PROPOSED GENERALIZED ESTIMATORS}

In this section, two generalized regression-cum-exponential type estimators are proposed by using the variance and mean respectively as the auxiliary variables in simple random sampling. The estimators are defined as

$t_{n 1}=\left[s_{y}^{2}+k_{1}\left(S_{z}^{2}-s_{z}^{2}\right)\right] \exp \left[\alpha_{1} \frac{S_{x}^{2}-s_{x}^{2}}{S_{x}^{2}+\left(\delta_{1}-1\right) s_{x}^{2}}\right]$

and

$t_{n 2}=\left[s_{y}^{2}+k_{2}\left(S_{z}^{2}-s_{z}^{2}\right)\right] \exp \left[\alpha_{2}\left(\frac{\bar{X}-\bar{x}}{\bar{X}+\left(\delta_{2}-1\right) \bar{x}}\right)\right]$,

where, $k_{1}, k_{2}, \delta_{1}, \delta_{2}\left(\delta_{1}, \delta_{2}>0\right)$ be the constants and need to be optimized for the expressions of minimum mean square errors for proposed estimators $\left(t_{n 1}, t_{n 2}\right)$. The generalized constants $\alpha_{1}$ and $\alpha_{2}$ can assume values $-1,0$ and 1 to generate many special cases of the suggested estimators.

\subsection{Bias and MSE for the Proposed Estimator-I $\left(t_{n I}\right)$}

In order to derive the expression of bias of the proposed estimator $t_{n 1}$, we may write (25) in terms of $e$ 's as

$$
t_{n 1}=\left[S_{y}^{2}\left(1+e_{0}\right)+k_{1}\left\{S_{z}^{2}-S_{z}^{2}\left(1+e_{2}\right)\right\}\right] \exp \left[\alpha\left(\frac{S_{x}^{2}-S_{x}^{2}\left(1+e_{1}\right)}{S_{x}^{2}+\left(\delta_{1}-1\right) S_{x}^{2}\left(1+e_{1}\right)}\right)\right] .
$$

Simplifying and applying Taylor series and ignoring the terms beyond the second order terms 
$t_{n 1} \approx\left[S_{y}^{2}+S_{y}^{2} e_{0}-k_{1} S_{z}^{2} e_{2}\right]\left[1-\frac{\alpha_{1}}{\delta_{1}} e_{1}+\frac{\alpha_{1}}{\delta_{1}} e_{1}^{2}-\frac{\alpha_{1}}{\delta_{1}^{2}} e_{1}^{2}+\frac{\alpha_{1}^{2}}{2 \delta_{1}^{2}} e_{1}^{2}\right]$

On simplification of (28), we have

$t_{n 1}-S_{y}^{2} \approx\left[-w_{1} S_{y}^{2} e_{1}+w_{1} S_{y}^{2} e_{1}^{2}\left(1-\frac{1}{\delta_{1}}+\frac{w_{1}}{2}\right)+S_{y}^{2} e_{0}-w_{1} S_{y}^{2} e_{1} e_{0}-k_{1} S_{z}^{2} e_{2}+w_{1} k_{1} S_{z}^{2} e_{1} e_{2}\right]$,

where

$\xi_{1}=\left(1-\frac{1}{\delta_{1}}+\frac{w_{1}}{2}\right)$

Simplifying and taking expectations of (29), we may get the expression of bias of the proposed estimator as

$\operatorname{Bias}\left(t_{n 1}\right) \approx \theta w_{1} S_{y}^{2}\left(\lambda_{040}-1\right)\left[\xi_{1}-{ }_{x} f_{220}+k_{1} \frac{S_{z}^{2}}{S_{y}^{2}}{ }_{x} f_{022}\right]$

In order to get the expression of $M S E$ of the proposed estimator $t_{n 1}$, we expand (27) using Tylor series up to the first order of approximation as

$t_{n 1}-S_{y}^{2} \approx S_{y}^{2} e_{0}-k_{1} S_{z}^{2} e_{2}-w_{1} S_{y}^{2} e_{1}$

By taking Square of (31) and ignoring the higher order terms

$$
\left(t_{n 1}-S_{y}^{2}\right)^{2} \approx\left[S_{y}^{4} e_{0}^{2}+k_{1}^{2} S_{z}^{4} e_{2}^{2}+w_{1}^{2} S_{y}^{4} e_{1}^{2}-2 k_{1} S_{y}^{2} S_{z}^{2} e_{0} e_{2}+2 k_{1} w_{1} S_{y}^{2} S_{z}^{2} e_{1} e_{2}-2 w_{1} S_{y}^{4} e_{0} e_{1}\right],
$$

by taking expectations on both sides of (32), we have the expression of MSE as

$$
\operatorname{MSE}\left(t_{n 1}\right) \approx \theta\left(\lambda_{400}-1\right)\left[S_{y}^{4}\left\{1+w_{1 x}^{2} f_{040}-2 w_{1 x} f_{220}\right\}+k_{1}^{2} S_{z x}^{4} f_{004}+2 S_{y}^{2} S_{z}^{2}\left\{w_{1 x} f_{022}-{ }_{x} f_{202}\right\}\right] .
$$

In order to minimize the expression of (33) with respect to " $w_{1}$ " and " $k_{1}$ " yield its optimum values as

$$
w_{1(o p t)}=\frac{1}{A}\left[{ }_{x} f_{220}-{ }_{x} f_{022} f_{202}\right] \quad \text { and } \quad k_{1(o p t)}=\frac{S_{y}^{2}}{S_{z}^{2}} \frac{1}{A}\left[A_{z} f_{202}-{ }_{z} f_{022 x} f_{220}+{ }_{z} f_{022 x} f_{022 z} f_{202}\right] .
$$

The expression of the minimized mean square error of $t_{n 1}$ after substituting the values of $w_{l(o p t)}$ and $k_{1(o p t)}$ obtained as,

$$
M S E_{\min }\left(t_{n 1}\right) \approx \theta S_{y}^{4}\left(\lambda_{400}-1\right) \frac{1}{A^{2}}\left[A^{2}+\left(R_{1 y} f_{004}+R_{2 y} f_{040}+2 R_{3 y} f_{022}\right)-2 A R_{4}\right]
$$

where,

$$
\begin{aligned}
& R_{1}=\left[{ }_{z} f_{202}^{2}+{ }_{z} f_{022 x}^{2} f_{220}^{2}-2{ }_{z} f_{202 z} f_{022 x} f_{220}\right], R_{2}=\left[{ }_{x} f_{220}^{2}+{ }_{x} f_{022 z}^{2} f_{202}^{2}-2{ }_{z} f_{202 x} f_{022 x} f_{220}\right], \\
& R_{3}=\left[{ }_{x} f_{220 z} f_{202}-{ }_{x} f_{220 z}^{2} f_{022}-{ }_{z} f_{202 x}^{2} f_{022}+{ }_{x} f_{022 x} f_{220 z} f_{202 z} f_{022}\right] .
\end{aligned}
$$




\subsection{Bias and $M S E$ for Proposed Estimator-II $\left(t_{n 2}\right)$}

In order to derive the expression of bias for the proposed estimators $t_{n 2}$, we may express (26) in terms of $e$ 's as,

$$
t_{n 2}=\left[S_{y}^{2}\left(1+e_{0}\right)+k_{2}\left\{S_{z}^{2}-S_{z}^{2}\left(1+e_{2}\right)\right\}\right] \exp \left[\alpha_{2}\left(\frac{\bar{X}-\bar{X}\left(1+e_{11}\right)}{\bar{X}+\left(\delta_{2}-1\right) \bar{X}\left(1+e_{11}\right)}\right)\right] .
$$

Simplifying and applying Taylor series on (35) up to the second order of approximation, we have

$$
\left(t_{n 2}-S_{y}^{2}\right) \approx\left[S_{y}^{2} e_{0}-w_{2} S_{y}^{2} e_{11}+w_{2} S_{y}^{2} e_{11}^{2}\left(1-\frac{1}{\delta_{2}}+\frac{w_{2}}{2}\right)-w_{2} S_{y}^{2} e_{0} e_{11}-k_{2} S_{z}^{2} e_{2}+k_{2} w_{2} S_{z}^{2} e_{2} e_{11}\right] .
$$

Applying expectation on (36), we have the final expression of the approximate bias of the proposed estimators $t_{n 2}$, as

$\operatorname{Bias}\left(t_{n 2}\right) \approx \theta S_{y}^{2} C_{x}^{2} w_{2}\left[\xi_{2}-\lambda_{210}+k_{2} \frac{S_{z}^{2}}{S_{y}^{2}} \lambda_{012}\right]$ where $\xi_{2}=\left(1-\frac{1}{\delta_{2}}+\frac{w_{2}}{2}\right)$

In order to get the expression of $M S E$ for the proposed estimator $t_{n 2}$, we expand (35) up to first order of approximation as

$t_{n 2}-S_{y}^{2}=S_{y}^{2} e_{0}-k_{2} S_{z}^{2} e_{2}-S_{y}^{2} w_{2} e_{11}$

By taking square and after simplifying the (38), we have

$$
\left(t_{n 2}-S_{y}^{2}\right)^{2}=\left[S_{y}^{4} e_{0}^{2}+k_{2}^{2} S_{z}^{4} e_{2}^{2}+S_{y}^{4} w_{2}^{2} e_{11}^{2}-2 k_{2} S_{y}^{2} S_{z}^{2} e_{0} e_{2}+2 k_{2} w_{2} S_{y}^{2} S_{z}^{2} e_{2} e_{11}-2 w_{2} S_{y}^{4} e_{0} e_{11}\right]
$$

By taking expectations of (39), we have

$$
\operatorname{MSE}\left(t_{n 2}\right) \approx \theta\left[\begin{array}{c}
S_{y}^{4}\left(\lambda_{400}-1\right)+k_{2}^{2} S_{z}^{4}\left(\lambda_{004}-1\right) \\
+2 k_{2} S_{y}^{2} S_{z}^{2}\left\{w_{2}\left(\lambda_{012}\right) C_{x}-\left(\lambda_{202}-1\right)\right\}-2 w_{2} S_{y}^{4}\left(\lambda_{210}\right) C_{x}+S_{y}^{4} w_{2}^{2} C_{x}^{2}
\end{array}\right] .
$$

In order to obtain the expression of minimum MSE, differentiating (40) with respect to " $w_{2}$ " and " $k$ " yield its optimum values

$$
w_{2(o p t)}=\frac{1}{B C_{x}}\left[\left(\lambda_{210}\right)-\left(\lambda_{012}\right)_{z} f_{202}\right] \text { and } k_{2(o p t)}=\frac{S_{y}^{2}}{S_{z}^{2}}\left[\left({ }_{z} f_{202}\right)-Z\left\{\left(\lambda_{210}\right)-\left(\lambda_{012}\right)\left({ }_{z} f_{202}\right)\right\}\right] \text {. }
$$

The final expression of the minimize $M S E$ of $t_{n 2}$ after substitute the values of $w_{2(\text { opt })}$ and $k_{2(\text { opt }}$ is,

$$
\operatorname{MSE}_{\min }\left(t_{n 2}\right) \approx \theta S_{y}^{4}\left[\left(\lambda_{400}-1\right) R_{1}+\frac{1}{B^{2}}\left\{Z\left(\lambda_{012}\right) R_{2}+\left(R_{3}-2\left(\lambda_{012}\right)^{2}\left(\lambda_{210}\right)^{2}\right)-2 B R_{3}\right\}\right]
$$


where

$$
\begin{aligned}
& R_{4}=\left[1+\left({ }_{y} f_{004}\right)\left({ }_{z} f_{202}\right)^{2}-2\left({ }_{y} f_{202}\right)\left({ }_{z} f_{202}\right)\right], R_{5}=\left[\left(\lambda_{210}\right)^{2}-\left(\lambda_{012}\right)^{2}\left({ }_{z} f_{202}\right)^{2}+2\left({ }_{z} f_{202}\right)\left(\lambda_{210}\right)\left(\lambda_{012}\right)\right], \\
& R_{6}=\left[\left(\lambda_{210}\right)^{2}+\left(\lambda_{012}\right)^{2}\left({ }_{z} f_{202}\right)^{2}-2\left({ }_{z} f_{202}\right)\left(\lambda_{210}\right)\left(\lambda_{012}\right)\right], \quad Z=\frac{\left(\lambda_{012}\right)}{B\left(\lambda_{004}-1\right)} .
\end{aligned}
$$

The details of populations are presented in Appendix Table-B3 in order to judge the performance of the proposed estimators over the competing estimators at optimum conditions. Some special cases of the proposed estimators are summarized in appendix-A

\section{EFFICIENCY COMPARISON OF PROPOSED ESTIMATORS WITH SOME EXISTING ESTIMATORS}

The efficiency comparisons of the proposed estimators with some relevant competing estimators are given as.

i. The proposed $\left(t_{n 1}\right)$ will be more precise estimator than the [1] estimator given in (3) if

$$
\begin{aligned}
& \operatorname{MSE}_{\text {min }}\left(t_{n 1}\right)<\operatorname{MSE}\left(t_{1}\right), \\
& { }_{x} f_{220 y} f_{220}+M_{1}<0 .
\end{aligned}
$$

ii. The proposed $\left(t_{n 1}\right)$ will be more precise estimator than the [4] estimator given in (9) if

$$
\begin{aligned}
& M S E_{\text {min }}\left(t_{n 1}\right)<\operatorname{MSE}\left(t_{4}\right), \\
& M_{1}\left(\lambda_{400}-1\right)-\frac{\lambda_{040}}{4}+\lambda_{220}-\frac{5}{4}<0 .
\end{aligned}
$$

iii. The proposed $\left(t_{n 1}\right)$ will be more precise estimator than the [9] estimator given in (19)

$$
\begin{aligned}
& \operatorname{MSE}_{\text {min }}\left(t_{n 1}\right)<\operatorname{MSE}\left(t_{9}\right), \\
& M_{1}\left(\lambda_{400}-1\right)+\frac{3\left(\lambda_{040}-1\right)}{16}\left(3-8_{x} f_{220}\right)<0 .
\end{aligned}
$$

iv. The proposed $\left(t_{n 2}\right)$ will be more precise estimator than the traditional regression estimator given in (13) if

$$
\begin{aligned}
& M S E_{\min }\left(t_{n 2}\right)<\operatorname{MSE}\left(t_{3}\right), \\
& \left(\lambda_{400}-1\right)\left(R_{1}-1\right)+\lambda_{210}^{2}<0 .
\end{aligned}
$$

v. The proposed $\left(t_{n 2}\right)$ will be more precise estimator than the [8] estimator given in (15) if 


$$
\begin{aligned}
& M S E_{\min }\left(t_{n 2}\right)<\operatorname{MSE}\left(t_{7}\right), \\
& \left(\lambda_{400}-1\right)\left(R_{1}-1\right)+\lambda_{210} C_{x}-0.25 C_{x}^{2}+M_{2}<0 .
\end{aligned}
$$

vi. The proposed $\left(t_{n 2}\right)$ will be more precise estimator than the [11] the estimator is given in (23) if

$$
\begin{aligned}
& M S E_{\min }\left(t_{n 2}\right)<\operatorname{MSE}\left(t_{11}\right), \\
& \left(\lambda_{400}-1\right)\left(R_{1}-1\right)+\lambda_{210}^{2}+M_{2}+P<0 .
\end{aligned}
$$

\section{NUMERICAL STUDY}

In this section, we set up two types of numerical studies in order to evaluate the performance of the estimators consider in this paper using three real populations. The judgment for the evaluation of suggested estimators are illustrated with traditional unbiased variance estimator. The amount of $A R B, M S E$ and percent relative efficiency ( $P R E$ 's) may be obtained by using the following mathematical formulas as

$$
\begin{aligned}
& A R B\left(t_{i}\right)=\frac{\left|\frac{1}{R} \sum_{i=1}^{R}\left(t_{i}-S_{y}^{2}\right)\right|}{S_{y}^{2}}, \\
& \operatorname{MSE}\left(t_{i}\right)=\frac{1}{R} \sum_{i=1}^{R}\left(t_{i}-S_{y}^{2}\right)^{2},
\end{aligned}
$$

and

$$
\operatorname{PRE}=\frac{\operatorname{Var}\left(t_{0}\right)}{\operatorname{MSE}\left(t_{*}\right)} \times 100,
$$

where ' $R$ ' $(R=20,000)$ is the total number of iterations and $t_{i}$ be the relevant estimators for $i^{\text {th }}$ sample. The performance of the proposed estimators depends on PRE, such that the value greater than one hundred indicates that the proposed estimators are more efficient than the usual variance estimator.

\subsection{Theoratical Study}

In this section, we evaluated the performance of our proposed estimators on three real populations using an empirical study and the results of PRE's are summarized in Table 1. The sources of populations along with the descriptions of study and auxiliary variables are given in Appendix-B.

Table 1. Percentage Relative Efficiencies of all the Estimators

\begin{tabular}{|l|l|l|l|}
\hline \multirow{2}{*}{ Estimators } & \multicolumn{3}{|c|}{ Populations } \\
\cline { 2 - 4 } & 1 & 2 & 3 \\
\hline$t_{0}=s_{y}^{2}$ & 100 & 100 & 100 \\
\hline$t_{1}$ & 41.5495 & 157.2156 & 89.4363 \\
\hline$t_{2}$ & 108.2613 & 180.2517 & 102.5925 \\
\hline$t_{3}$ & 100.0395 & 138.2660 & 109.2543 \\
\hline$t_{4}$ & 74.6328 & 140.2744 & 91.1857 \\
\hline$t_{6}$ & 108.2613 & 180.2517 & 102.5925 \\
\hline$t_{7}$ & 99.6919 & 80.9687 & 93.8899 \\
\hline
\end{tabular}




\begin{tabular}{|l|l|l|l|}
\hline$t_{9}$ & 61.1047 & 179.5361 & 96.6753 \\
\hline$t_{10}$ & 100.0395 & 109.9673 & 102.6304 \\
\hline$t_{11}$ & 120.5324 & 183.3319 & 109.2887 \\
\hline$t_{n 1}$ (proposed) & 151.3692 & 323.9974 & 139.9441 \\
\hline$t_{n 2}$ (proposed) & 159.4911 & 348.4204 & 149.7324 \\
\hline
\end{tabular}

The results of the empirical study are presented in Table1 indicate that our proposed estimators $t_{n 1}, t_{n 2}$ performed better and found to be the most efficient estimators as compared to [1] $t_{1}, t_{2}$ and $t_{3}$ estimators, [4] estimator $t_{4},[8]$ estimator $t_{5}$, [9] estimators $t_{9},[10]$ estimator $t_{10}$ and [11] estimator $t_{11}$.

\subsection{Simulation Study}

In this simulation we consider six sample sizes $n=8,11,14,17,20$ and 23 to evaluate the performance of proposed estimators and the results are summarized in Table 2 and Table 3 respectively. The following steps have been used to compute the $A R B$ 's and PRE's by using the $R$-Language software.

Step1 From population 3, Twenty thousand samples of size $\mathrm{n}$ we selected using SRSWOR.

Step2 Using the sample of step 1, Twenty thousand values of all the estimators are obtained from each sample size to achieve the efficiency in the estimation.

Step3 The $A R B$, and $P R E$ of all the considered estimators are computed using the formula given in eq.(42) and eq.(44) . 
Table 2. Absolute relative bias of all the estimators for different sample sizes

\begin{tabular}{|l|l|l|l|l|l|l|}
\hline \multirow{2}{*}{ Estimators } & \multicolumn{6}{|c|}{ Sample size } \\
\cline { 2 - 7 } & 8 & 11 & 14 & 17 & 20 & 23 \\
\hline$t_{0}=s_{y}^{2}$ & 0.6456 & 0.3736 & 0.5064 & 0.4935 & 0.3325 & 0.2009 \\
\hline$t_{1}$ & 0.3917 & 0.1893 & 0.2417 & 0.4295 & 0.1559 & 0.0258 \\
\hline$t_{2}$ & 0.5222 & 0.3064 & 0.4032 & 0.4802 & 0.2706 & 0.1504 \\
\hline$t_{3}$ & 0.6164 & 0.3903 & 0.4706 & 0.4804 & 0.3186 & 0.1842 \\
\hline$t_{4}$ & 0.5387 & 0.2879 & 0.3902 & 0.4611 & 0.2498 & 0.1101 \\
\hline$t_{6}$ & 0.5779 & 0.3214 & 0.4340 & 0.4744 & 0.2823 & 0.1480 \\
\hline$t_{7}$ & 0.6205 & 0.3953 & 0.4625 & 0.4524 & 0.3112 & 0.1592 \\
\hline$t_{9}$ & 0.4652 & 0.2386 & 0.3159 & 0.4453 & 0.2028 & 0.0679 \\
\hline$t_{10}$ & 0.6333 & 0.3846 & 0.4849 & 0.4728 & 0.3219 & 0.1799 \\
\hline$t_{11}$ & 0.6268 & 0.3749 & 0.4799 & 0.4762 & 0.3182 & 0.1795 \\
\hline$t_{n 1}$ (Proposed) & 0.6839 & 0.5362 & 0.1652 & 0.5181 & 0.0319 & 0.0087 \\
\hline$t_{n 2}$ (Proposed) & 0.6526 & 0.5382 & 0.0618 & 0.5017 & 0.0303 & 0.0499 \\
\hline
\end{tabular}

Table 3: Percentage relative efficiencies for all the estimators of different sample sizes

\begin{tabular}{|l|l|l|l|l|l|l|}
\hline \multirow{2}{*}{ Estimators } & \multicolumn{6}{|c|}{ Sample size } \\
\cline { 2 - 7 } & 8 & 11 & 14 & 17 & 20 & 23 \\
\hline$t_{0}=s_{y}^{2}$ & 100 & 100 & 100 & 100 & 100 & 100 \\
\hline$t_{1}$ & 68.8873 & 76.4613 & 76.94706 & 76.5429 & 77.6677 & 77.3710 \\
\hline$t_{2}$ & 102.9675 & 103.2390 & 103.2839 & 102.9013 & 102.7457 & 102.4545 \\
\hline$t_{3}$ & 102.1620 & 102.2128 & 102.2547 & 102.2111 & 102.2037 & 102.1727 \\
\hline$t_{4}$ & 101.3425 & 100.2903 & 98.9985 & 97.5976 & 97.2824 & 96.5506 \\
\hline$t_{6}$ & 102.8320 & 102.1749 & 101.3834 & 100.5272 & 100.2806 & 99.8629 \\
\hline$t_{7}$ & 105.4710 & 104.5317 & 104.0685 & 103.5067 & 103.2695 & 103.0139 \\
\hline$t_{9}$ & 87.11435 & 90.0978 & 89.3678 & 88.2704 & 88.5735 & 87.9722 \\
\hline$t_{10}$ & 103.0179 & 102.5238 & 102.2670 & 101.9794 & 101.8474 & 101.7150 \\
\hline$t_{11}$ & 102.9033 & 102.4082 & 102.1174 & 101.8063 & 101.6656 & 101.5269 \\
\hline$t_{n 1}$ (Proposed) & 132.7014 & 135.3150 & 139.5052 & 141.5388 & 141.2783 & 141.3261 \\
\hline$t_{n 2}$ (Proposed) & 135.7227 & 137.8522 & 141.5803 & 143.2343 & 142.7709 & 142.3033 \\
\hline
\end{tabular}

\section{CONCLUSION}

The results illustrated in Section-5 which are summarized in Table 2,Table 3 and Table 4 respectively shows that the proposed estimators are more efficient than the other estimators considered in this paper. The results obtained from empirical study as shown in Table 1 found to be more superior then the other existing estimators. From the results of simulation study, it is visibly acquired that the values of $A R B$ for all the estimators tend to zero and the PRE increases by increasing the sample sizes as shown in Table 2 and Table 3 respectively. Hence the proposed estimators are most efficient and useful for the estimation of finite population variance. Some special cases of proposed estimators are given in Appendix-A. Source of populations, description of variables and results of parameters are given in Appendix-B. 


\section{ACKNOWLEDGEMENT}

The authors are thankful to the anonymous raferees for the improvement of the paper. The first author is greatful to Syed Wasim Abbas for the final editing of the paper

\section{CONFLICTS OF INTEREST}

No conflict of interst was declared by the authors.

\section{REFERENCES}

[1] Isaki, C.T., "Variance estimation using auxiliary information", Journal of the American Statistical Association, 78(381): 117-123, (1983).

[2] Prasad, B., Singh, H.P., "Some improved ratio-type estimators of finite population variance in sample surveys", Communications in Statistics-Theory and Methods, 19(3): 1127-1139, (1990).

[3] Prasad, B., Singh, H.P., "Unbiased estimators of finite population variance using auxiliary information in sample surveys", Communications in Statistics-Theory and Methods, 21(5): 13671376, (1992).

[4] Singh, R., Chauhan, P., Sawan, N., Smarandache, F., "Improved exponential estimator for population variance using two auxiliary variables", Italian Journal of Pure and Applied Mathematics, 28: 101-108, (2011).

[5] Yadav, S.K., Kadilar, C., "Improved Exponential Type Ratio Estimator of Population Variance", Revista Colombiana de Estadística,36(1): 145-152, (2013).

[6] Upadhyaya, L.N., Singh, H.P., "An estimator for population variance that utilizes the kurtosis of an auxiliary variable in sample surveys", Vikram Mathematical Journal, 19(1):14-17, (1999).

[7] Jhajj, H.S., Sharma, M.K., Grover, L.K., "An efficient class of chain estimators of population variance under sub-sampling scheme", Journal of the Japan Statistical Society, 35(2): 273-286, (2005).

[8] Asghar, A., Sanaullah, A., Hanif, M.,“Generalized exponential type estimator for population variance in survey sampling”, Revista Colombiana de Estadística, 37(1): 213-224,(2014).

[9] Singh, H.P., Singh, A.K., Solanki, R.S., "Estimation of finite population variance using auxiliary information in sample surveys", Statistica, 74(1): 95-112, (2014).

[10] Ahmed, A., Singh, R. V. K., "Improved exponential type estimators for estimating population variance in survey sampling", International Journal of Advance Research, IJOAR .org, 33(1): 1-16, (2015).

[11] Gupta, S., Shabbir, J., "Variance estimation in simple random sampling using auxiliary information", Hacettepe Journal of Mathematics and Statistics, 37(1): 57-67, (2008).

[12] Cekim, H.O., Kadilar, C., "Generalized family of estimators via two auxiliary variables for population variance", Journal of Reliability and Statistical Studies, 11(1): 67-81, (2018).

[13] Grover, L.K., "A correction note on improvement in variance estimation using auxiliary information", Communications in Statistics-Theory and Methods, 39(5): 753-764, (2010). 
[14] Jhajj, H.S., Lata, K., "A family of difference-cum-exponential type estimators for estimating the population variance using auxiliary information in sample surveys", Journal of Mathematics and Statistics Sciences, 3(4): 10-14, (2015).

[15] Kadilar, C., Cingi, H., "Improvement in variance estimation using auxiliary information", Hacettepe Journal of Mathematics and Statistics, 35(1): 111-115, (2006).

[16] Khan, M., Shabbir, J., "A ratio type estimator for the estimation of population variance using quartiles of an auxiliary variable", Journal of Statistics Applications \& Probability, 2(3): 319-325, (2013).

[17] Maqbool, S., Javaid, S., "Variance estimation using linear combination of tri-mean and quartile average", American Journal of Biological and Environmental Statistics, 3(1): 5-9, (2017).

[18] Subramani, J., Kumarapandiyan, G., "Variance estimation using median of the auxiliary variable", International Journal of Probability and Statistics, 1(3): 36-40, (2012).

[19] Subramani, J., Kumarapandiyan, G., "Estimation of variance using known coefficient of variation and median of an auxiliary variable", Journal of Modern Applied Statistical Methods, 12(1):58- 64, (2013).

[20] Singh, H.P., Solanki, R.S., "A new procedure for variance estimation in simple random sampling using auxiliary information”, Statistical Papers, 54(2): 479-497, (2013).

[21] Yadav, S.K., Misra, S., Mishra, S.S., "Efficient estimator for population variance using auxiliary variable", American Journal of Operational Research, 6(1): 9-15, (2016).

[22] Shabbir, J., Gupta, S., "A note on generalized exponential type estimator for population variance in survey sampling”, Revista Colombiana de Estadística, 38(2): 385-397, (2015).

[23] Singh, H.P., Espejo, M.R., "On linear regression and ratio-product estimation of a finite population mean”, Journal of the Royal Statistical Society: Series D (The Statistician), 52(1): 59-67, (2003).

[24] Cochran, W.G., "Sampling Techniques”, New York: John Wiley and Sons,(1977).

[25] Gujarati, D.N., "Basic econometrics", Tata McGraw-Hill Education, (2009).

[26] Sukhatme, P.V., Sukhatme, B.V., "Sampling Theory of Surveys with Applications", Asia Publishing House, New Delhi, (1970). 


\section{APPENDIX-A}

The proposed estimators $t_{n 1}$ and $t_{n 2}$ may produce many special cases by using different values $\alpha_{1}, \alpha_{2}, k_{1}, k_{2}$ $\delta_{1}$ and $\delta_{2}$. Some of them are listed below.

Table A1. Some Special Cases of Proposed Estimators

\begin{tabular}{|c|c|c|c|c|c|c|c|}
\hline$t_{n 1}$ & $t_{n 2}$ & $\alpha_{1}$ & $\alpha_{2}$ & $k_{1}$ & $k_{2}$ & $\delta_{1}$ & $\delta_{2}$ \\
\hline$t_{0}=s_{y}^{2}$ & $t_{0}=s_{y}^{2}$ & 0 & 0 & 0 & 0 & 1 & 1 \\
\hline$t_{n 1}^{1}=s_{y}^{2} \exp \left[\frac{S_{x}^{2}-s_{x}^{2}}{S_{x}^{2}}\right]$ & $t_{n 2}^{1}=s_{y}^{2} \exp \left[\frac{\overline{\mathrm{X}}-\bar{x}}{\bar{X}}\right]$ & 1 & 1 & 0 & 0 & 1 & 1 \\
\hline$t_{n 1}^{2}=s_{y}^{2} \exp \left[\frac{S_{x}^{2}-s_{x}^{2}}{S_{x}^{2}+s_{x}^{2}}\right]$ & $t_{n 2}^{2}=s_{y}^{2} \exp \left[\frac{\bar{X}-\bar{x}}{\bar{X}+\bar{x}}\right]$ & 1 & 1 & 0 & 0 & 2 & 2 \\
\hline$t_{n 3}^{3}=s_{y}^{2} \exp \left[\frac{S_{x}^{2}-s_{x}^{2}}{S_{x}^{2}+\left(\delta_{\text {lopt }}-1\right) s_{x}^{2}}\right]$ & $\hat{t}_{n 2}^{3}=s_{,}^{2} \exp \left[\frac{\bar{X}-\bar{x}}{\left.\bar{X}+\left(\delta_{2(x)}\right)-1\right) \bar{x}}\right]$ & 1 & 1 & 0 & 0 & $\delta_{1(o p t)}$ & $\delta_{2 o p t}$ \\
\hline$t_{n 1}^{4}=s_{y}^{2} \exp \left[\frac{s_{x}^{2}-S_{x}^{2}}{S_{x}^{2}}\right]$ & $t_{n 2}^{4}=s_{y}^{2} \exp \left[\frac{\bar{x}-\bar{X}}{\bar{X}}\right]$ & -1 & -1 & 0 & 0 & 1 & 1 \\
\hline$t_{n 1}^{5}=s_{y}^{2} \exp \left[\frac{s_{x}^{2}-S_{x}^{2}}{S_{x}^{2}+s_{x}^{2}}\right]$ & $t_{n 2}^{5}=s_{y}^{2} \exp \left[\frac{\bar{x}-\bar{X}}{\bar{X}+\bar{x}}\right]$ & -1 & -1 & 0 & 0 & 2 & 2 \\
\hline$t_{n 1}^{6}=s_{y}^{2}+b_{y z}\left(\mathrm{~S}_{z}^{2}-s_{z}^{2}\right)$ & $t_{n 2}^{6}=s_{y}^{2}+b_{y z}\left(S_{z}^{2}-s_{z}^{2}\right)$ & 0 & 0 & $b_{y z}$ & $b_{y z}$ & 1 & 1 \\
\hline$t_{n 1}^{7}=\left[s_{y}^{2}+b_{y z}\left(S_{z}^{2}-s_{z}^{2}\right)\right] \exp \left[\frac{S_{x}^{2}-s_{x}^{2}}{S_{x}^{2}}\right]$ & $t_{n 2}^{7}=\left[s_{y}^{2}+b_{y z}\left(S_{z}^{2}-s_{z}^{2}\right)\right] \exp \left[\frac{\bar{X}-\bar{x}}{\bar{X}}\right]$ & 1 & 1 & $b_{y z}$ & $b_{y z}$ & 1 & 1 \\
\hline$t_{n 1}^{8}=\left[s_{y}^{2}+b_{y z}\left(S_{z}^{2}-s_{z}^{2}\right)\right] \exp \left[\frac{s_{x}^{2}-S_{x}^{2}}{S_{x}^{2}}\right]$ & $t_{n 2}^{8}=\left[s_{y}^{2}+b_{y z}\left(S_{z}^{2}-s_{z}^{2}\right)\right] \exp \left[\frac{\bar{x}-\bar{X}}{\bar{X}}\right]$ & -1 & -1 & $b_{y z}$ & $b_{y z}$ & 1 & 1 \\
\hline$t_{n 1}^{9}=\left[s_{y}^{2}+\left(S_{z}^{2}-s_{z}^{2}\right)\right] \exp \left[\frac{S_{x}^{2}-s_{x}^{2}}{S_{x}^{2}}\right]$ & $t_{n 2}^{9}=\left[s_{y}^{2}+\left(S_{z}^{2}-s_{z}^{2}\right)\right] \exp \left[\frac{\bar{X}-\bar{x}}{\bar{X}}\right]$ & 1 & 1 & 1 & 1 & 1 & 1 \\
\hline$t_{n 1}^{10}=\left[s_{y}^{2}+\left(S_{z}^{2}-s_{z}^{2}\right)\right] \exp \left[\frac{s_{x}^{2}-S_{x}^{2}}{S_{x}^{2}}\right]$ & $t_{n 2}^{10}=\left[s_{y}^{2}+\left(S_{z}^{2}-s_{z}^{2}\right)\right] \exp \left[\frac{\bar{x}-\bar{X}}{\bar{X}}\right]$ & -1 & -1 & 1 & 1 & 1 & 1 \\
\hline$t_{n 1}^{11}=\left[s_{y}^{2}+\left(S_{z}^{2}-s_{z}^{2}\right)\right] \exp \left[\frac{S_{x}^{2}-s_{x}^{2}}{S_{x}^{2}+s_{x}^{2}}\right]$ & $t_{n 2}^{11}=\left[s_{y}^{2}+\left(S_{z}^{2}-s_{z}^{2}\right)\right] \exp \left[\frac{\bar{X}-\bar{x}}{\bar{X}+\bar{x}}\right]$ & 1 & 1 & 1 & 1 & 2 & 2 \\
\hline$t_{n 1}^{12}=\left[s_{y}^{2}+\left(S_{z}^{2}-s_{z}^{2}\right)\right] \exp \left[\frac{s_{x}^{2}-S_{x}^{2}}{S_{x}^{2}+s_{x}^{2}}\right]$ & $t_{n 2}^{12}=\left[s_{y}^{2}+\left(S_{z}^{2}-s_{z}^{2}\right)\right] \exp \left[\frac{\bar{x}-\bar{X}}{\bar{X}+\bar{x}}\right]$ & -1 & -1 & 1 & 1 & 2 & 2 \\
\hline$t_{n 1}^{13}=\left[s_{y}^{2}+b_{y z}\left(S_{z}^{2}-s_{z}^{2}\right)\right] \exp \left[\frac{S_{x}^{2}-s_{x}^{2}}{S_{x}^{2}+s_{x}^{2}}\right]$ & $t_{n 2}^{13}=\left[s_{y}^{2}+b_{y z}\left(S_{z}^{2}-s_{z}^{2}\right)\right] \exp \left[\frac{\bar{X}-\bar{x}}{\bar{X}+\bar{x}}\right]$ & 1 & 1 & $b_{y z}$ & $b_{y z}$ & 2 & 2 \\
\hline$t_{n 1}^{14}=\left[s_{y}^{2}+b_{y z}\left(S_{z}^{2}-s_{z}^{2}\right)\right] \exp \left[\frac{s_{x}^{2}-S_{x}^{2}}{S_{x}^{2}+s_{x}^{2}}\right]$ & $t_{n 2}^{14}=\left[s_{y}^{2}+b_{y z}\left(S_{z}^{2}-s_{z}^{2}\right)\right] \exp \left[\frac{\bar{x}-\bar{X}}{\bar{X}+\bar{x}}\right]$ & -1 & -1 & $b_{y z}$ & $b_{y z}$ & 2 & 2 \\
\hline$t_{n 1}^{15}=\left[s_{y}^{2}+b_{y z}\left(S_{z}^{2}-s_{z}^{2}\right)\right] \exp \left[\frac{S_{x}^{2}-s_{x}^{2}}{S_{x}^{2}+\left(\delta_{\text {lopt }}-1\right) s_{x}^{2}}\right]$ & $t_{n 2}^{15}=\left[s_{y}^{2}+b_{y z}\left(S_{z}^{2}-s_{z}^{2}\right)\right] \exp \left[\frac{\overline{\mathrm{X}}-\bar{x}}{\bar{X}+\left(\delta_{20 p t}-1\right) \bar{x}}\right]$ & 1 & 1 & $b_{y z}$ & $b_{y z}$ & $\delta_{1(o p t)}$ & $\delta_{2 o p t}$ \\
\hline$t_{n 1}^{16}=\left[s_{y}^{2}+b_{y z}\left(S_{z}^{2}-s_{z}^{2}\right)\right] \exp \left[\frac{s_{x}^{2}-S_{x}^{2}}{S_{x}^{2}+\left(\delta_{\text {lopt }}-1\right) s_{x}^{2}}\right]$ & $t_{n 2}^{16}=\left[s_{y}^{2}+b_{y z}\left(S_{z}^{2}-s_{z}^{2}\right)\right] \exp \left[\frac{\bar{X}-\bar{X}}{\bar{X}+\left(\delta_{20 p t}-1\right) \bar{x}}\right]$ & -1 & 1 & $b_{y z}$ & $b_{y z}$ & $\delta_{1(o p t)}$ & $\delta_{2 o p t}$ \\
\hline$t_{n 1}^{17}=s_{y}^{2} \exp \left[\frac{S_{x}^{2}-s_{x}^{2}}{S_{x}^{2}+(a-1) s_{x}^{2}}\right]$ & $t_{n 2}^{17}=s_{y}^{2} \exp \left[\frac{\bar{X}-\bar{x}}{\bar{X}+(a-1) \bar{x}}\right]$ & 1 & 1 & 0 & 0 & $\mathrm{a}$ & $\mathrm{a}$ \\
\hline
\end{tabular}




\section{APPENDIX-B}

Table-B1

\begin{tabular}{|l|l|}
\hline Population & Sources of Population \\
\hline 1 & Gujarati(2009, Pg 406) \\
\hline 2 & Sukhatme and Sukhatme (1970, pg 185) \\
\hline 3 & Cochran(1977,pg 34) \\
\hline
\end{tabular}

Table- B2

\begin{tabular}{|l|l|l|l|}
\hline Population & Y & X & Z \\
\hline 1 & $\begin{array}{l}\text { Average Miles per } \\
\text { gallon }\end{array}$ & $\begin{array}{l}\text { Top, speed miles per } \\
\text { Hour }\end{array}$ & Engine horsepower \\
\hline 2 & $\begin{array}{l}\text { The area under wheat in } \\
\text { acres during 1937 }\end{array}$ & $\begin{array}{l}\text { The area under wheat in } \\
\text { acres during 1936 }\end{array}$ & $\begin{array}{l}\text { The area under wheat } \\
\text { in acres during 1931 }\end{array}$ \\
\hline 3 & Food cost & Size & Income \\
\hline
\end{tabular}

Table B3 Results for Population Parameters

\begin{tabular}{|l|l|l|l|}
\hline \multirow{2}{*}{ parameters } & \multicolumn{3}{|l|}{ Populations } \\
\cline { 2 - 4 }$N$ & 1 & 2 & 3 \\
\hline$n$ & 21 & 34 & 33 \\
\hline $\bar{Y}$ & 33.7111 & 201.4118 & 27.4909 \\
\hline $\bar{X}$ & 112.4568 & 218.4118 & 3.7272 \\
\hline $\bar{Z}$ & 30.9876 & 765.3529 & 72.5455 \\
\hline$C_{y}$ & 0.2984 & 0.7555 & 0.3685 \\
\hline$C_{x}$ & 0.1256 & 0.7678 & 0.4095 \\
\hline$C_{z}$ & 0.2635 & 0.6169 & 0.1458 \\
\hline$\rho_{y x}$ & -0.6883 & 0.9299 & 0.4328 \\
\hline$\rho_{y z}$ & -0.9035 & 0.8992 & 0.2522 \\
\hline$\rho_{x z}$ & 0.6788 & 0.8308 & -0.0660 \\
\hline$\lambda_{400}$ & 3.5699 & 5.1118 & 5.5509 \\
\hline$\lambda_{040}$ & 6.7365 & 4.7293 & 2.3154 \\
\hline$\lambda_{004}$ & 2.4758 & 3.9524 & 2.0780 \\
\hline$\lambda_{220}$ & 2.0606 & 3.61287 & 1.3889 \\
\hline$\lambda_{202}$ & 2.1238 & 3.8722 & 2.2186 \\
\hline$\lambda_{022}$ & 1.9995 & 2.9763 & 1.4466 \\
\hline$\lambda_{110}$ & 0.0319 & 1.0668 & 0.6209 \\
\hline$\lambda_{201}$ & -0.0647 & 1.0686 & 0.5422 \\
\hline$\lambda_{012}$ & 0.4349 & 0.7541 & 0.2337 \\
\hline$\lambda_{021}$ & 0.8782 & 0.7404 & 0.2643 \\
\hline$\lambda_{030}$ & 1.8547 & 1.3184 & 0.5683 \\
\hline & & & \\
\hline
\end{tabular}

